\title{
Coupling Programs and Visualization for Machine Knitting
}

\author{
Tianhong Catherine $\mathrm{Yu}$ \\ James McCann \\ tianhony@andrew.cmu.edu \\ jmccann@cs.cmu.edu \\ Carnegie Mellon University
}

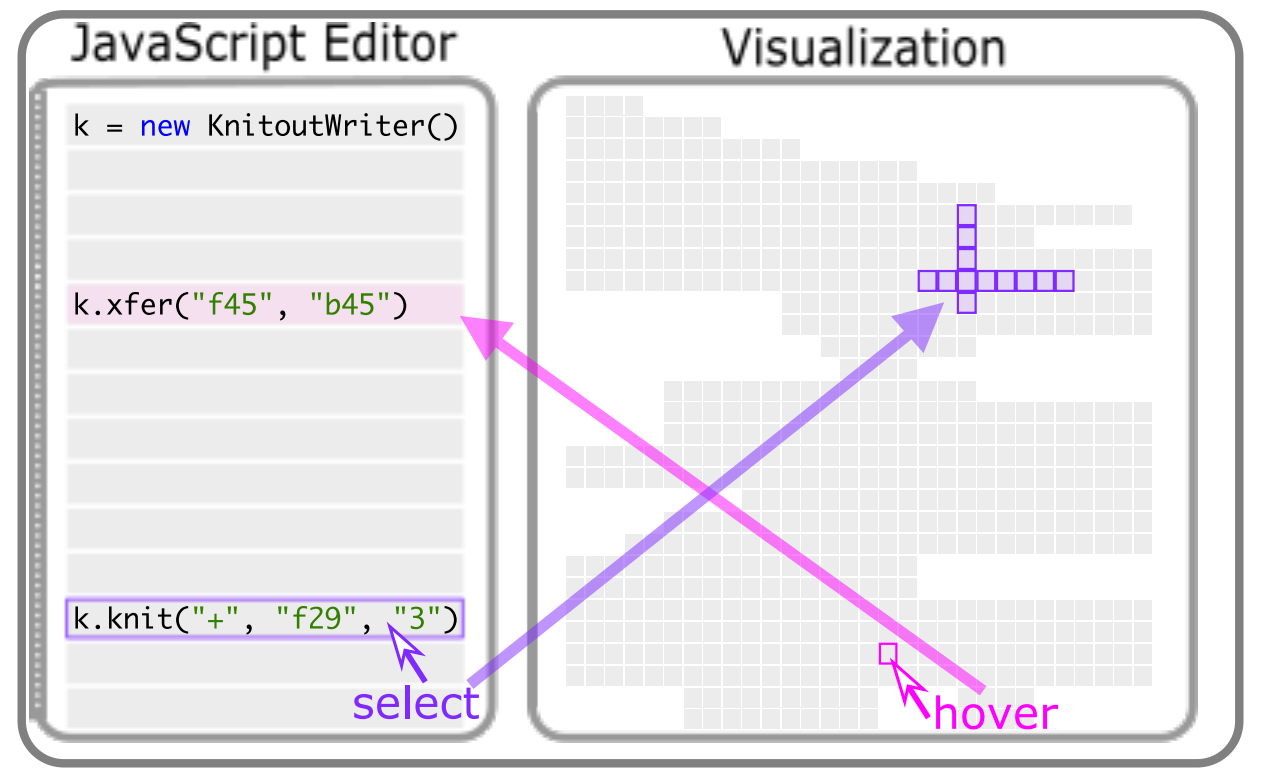

Figure 1: Our interface for knit programming includes a coupled visualization that allows users to select code to see all of the knit stitches it created, and hover any knit operation to see the line of code that produced it.

\begin{abstract}
To effectively program knitting machines, like any fabrication machine, users must be able to place the code they write in correspondence with the output the machine produces. This mapping is used in the code-to-output direction to understand what their code will produce, and in the output-to-code direction to debug errors in the finished product. In this paper, we describe and demonstrate an interface that provides two-way coupling between highor low-level knitting code and a topological visualization of the knitted output. Our system allows the user to locate the knitting machine operations generated by any selected code, as well as the code that generates any selected knitting machine operation. This link between the code and visualization has the potential to reduce the time spent in design, implementation, and debugging phases,
\end{abstract}

Permission to make digital or hard copies of all or part of this work for personal or classroom use is granted without fee provided that copies are not made or distributed for profit or commercial advantage and that copies bear this notice and the full citation on the first page. Copyrights for components of this work owned by others than the author(s) must be honored. Abstracting with credit is permitted. To copy otherwise, or republish, to post on servers or to redistribute to lists, requires prior specific permission and/or a fee. Request permissions from permissions@acm.org.

SCF '20, November 5-6, 2020, Virtual Event, USA

(C) 2020 Copyright held by the owner/author(s). Publication rights licensed to ACM ACM ISBN 978-1-4503-8170-3/20/11 . \$15.00

https://doi.org/10.1145/3424630.3425410 and save material costs by catching errors before actually knitting the object. We show examples of patterns designed using our tool and describe common errors that the tool catches when used in an academic lab setting and an undergraduate course.

\section{CCS CONCEPTS}

- Applied computing $\rightarrow$ Computer-aided manufacturing; • Computing methodologies $\rightarrow$ Graphics systems and interfaces; $\bullet$ Human-centered computing $\rightarrow$ Visualization.

\section{KEYWORDS}

machine knitting, knit programming, two-view interface, coupled visualization

\section{ACM Reference Format:}

Tianhong Catherine Yu and James McCann. 2020. Coupling Programs and Visualization for Machine Knitting. In Symposium on Computational Fabrication (SCF '20), November 5-6, 2020, Virtual Event, USA. ACM, New York, NY, USA, 10 pages. https://doi.org/10.1145/3424630.3425410

\section{INTRODUCTION}

Computer-aided design (CAD) tools and computational fabrication technologies offer many possibilities for people who want to make physical objects, but there still exists a divide between digital and 


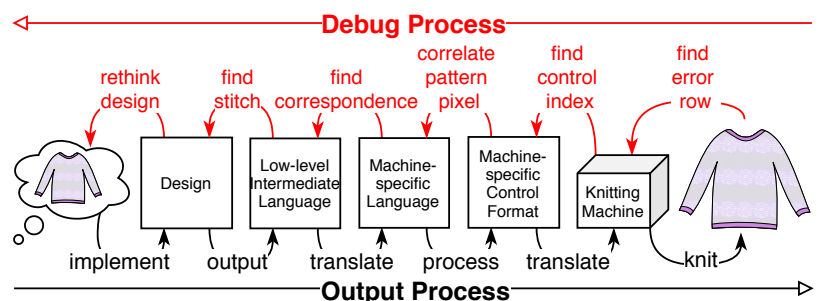

Figure 2: The complex design-system-to-knitted-output pipeline in current machine knitting design systems makes it difficult to correlate output stitches and input instructions.

physical manipulation [Gulay and Lucero 2019]. Throughout the cycle of ideation, design, implementation, and fabrication, users must draw correspondences between their digital inputs and the millions of operations executed by a physical output device. A clear and obvious relationship between the computer program and the expected output can make every stage of the process easier. Conversely, an unclear or complex relationship can complicate all stages of the design process, hinder the user in diagnosing errors, and also waste material.

Unfortunately, current high-level machine knitting design and fabrication workflows do not do a good job of maintaining correspondences between design primitives and machine actions. For example, with recent high-level design tools in research (Figure 2): users edit patterns visually in 3D [Narayanan et al. 2019; Popescu et al. 2018] or with high-level primitives [Kaspar et al. 2019; McCann et al. 2016]; output the pattern to a low-level intermediate language [McCann 2017]; use a utility to translate the low-level language into a manufacturer-specific language (e.g., Shima Seiki's KnitPaint); process this language into a machine-specific control format (e.g., a '.000' file for an SWG091N2 knitting machine); and finally execute the control format on a knitting machine to produce the knitted output. This deep pipeline becomes a problem when users encounter errors in later stages (e.g., the actual knitting). These errors are hard to match to the original input code or model, and therefore require extensive experience with the software (or arduous trial-and-error) to fix.

In this paper, we demonstrate an interface that provides realtime, two-way coupling between high-level knitting code and a topological visualization of the knitted output (Figure 1). Our tool lets users locate the knitting machine operations generated by any selected code, as well as the code that generates any selected knitting machine operations. This means that users can rapidly connect knitting errors to high-level code errors and fix them. We show examples of patterns designed using our tool and describe common errors that the tool catches when used in an academic lab setting and an undergraduate course. We invite interested readers to try the tool, which available via a git repository hosted at https: //github.com/textiles-lab/knitout-live-visualizer.

\section{BACKGROUND}

Live Programming for Computational Fabrication. Unlike live coding, an audiovisual performance practice which has been adapted

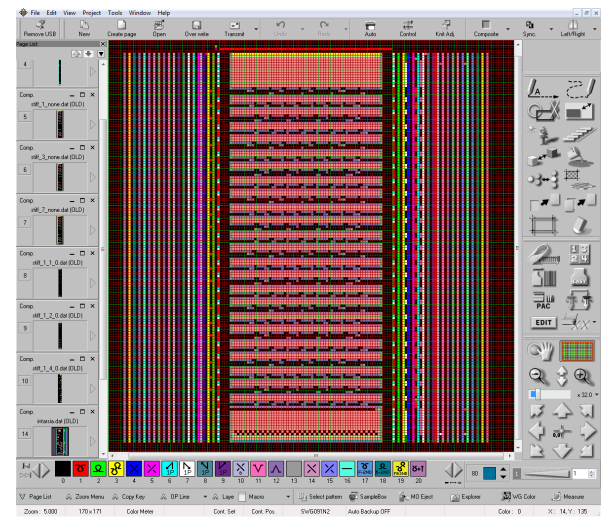

Figure 3: Lace Example in the KnitPaint pixel based programming interface.

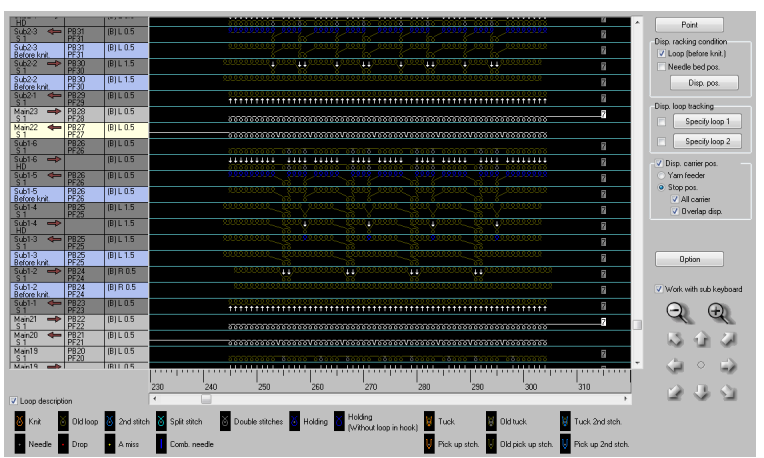

Figure 4: Lace example in the KnitPaint loop view. This view shows the state of the needle bed after every construction step, and can associate loops with the instructions that formed them.

for fabrication [Willis et al. 2010], our system offers a live programming experience. Live programming is the direct construction, manipulation, and visualization of a program's run-time state [Swift et al. 2013]. It is widely adopted in 3D modelling [Agrawal et al. 2014; Autodesk 2018; Kintel and Wolf 2017] and laser cutting [Freshour and Henty 2016]. It is also used in prototyping digital fabrication workflows [Tran O'Leary and Peek 2019] and carpentry [Stehling et al. 2017; Wu et al. 2019] to fill in the gap between CAD and CAM. With our system, users see a visualization of the knitted output as they program the knitting machine without actually fabricating the output.

Real-time Feedback and Visualization in Machine Knitting Programming. Researchers are excited about machine knitting for many reasons. Knitted fabric has the benefit of being flexible, lightweight, and breathable, but the design and fabrication process still remains rather complex. In order to reduce the number of iteration cycles in the design process, there has been work on creating a direct link between design (by, e.g., generated knitting patterns from images [Karmon et al. 2018]) and manufacturability. Real-time feedback is another way to facilitate the process. Visual knit editors 


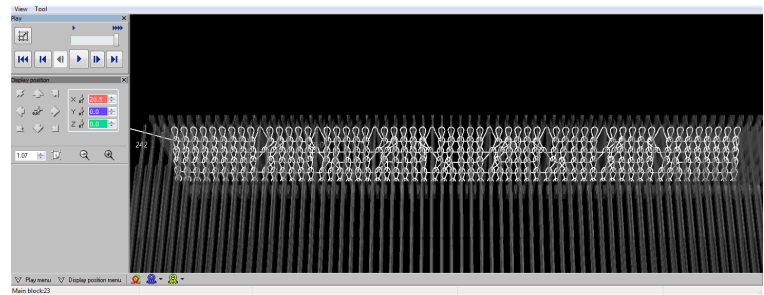

Figure 5: Lace example in KnitPaint's simulation window. This view animates the machine's operation and includes a physical yarn simulation of the most recent few rows of knitting. Note that this simulation takes 5-10 seconds per row of knitting - minutes or hours for a whole pattern - so is generally impractical as a debugging tool.

seek to highlight errors [Kaspar et al. 2019], or prevent the user from making edits that introduce errors [Narayanan et al. 2019]. Our system offers similar real-time visual feedback for users using textual programming for machine knitting. Our visualization is similar to the "string diagrams" in previous work [Suzuki et al. 2000].

Commercial knit programming systems such as Shima Seiki's KnitPaint [Shima Seiki 2011] (Figure 3) and Stolls's M1plus [Stoll 2011] use pixel-based programming, which itself serves as a sort of visualization - albeit one with a steep learning curve and a focus on machine operation rather than knit output. These commercial knit programs also include debugging and simulation tools including hand-coded consistency checks to catch common errors; control simulations which provide visualization of machine state and fabrication steps (Figure 4); and even, at least in the case of Shima Seiki's KnitPaint software, physically-based 3D yarn simulation for topology tracking (Figure 5). Our system provides a visualization with the topological fidelity of the latter and the speed of the former, and integrates this visualization into a high-level programming environment instead of with a machine-specific language.

Two-view Interfaces. A two-view interface takes advantage of both WYSIWYG and textual/descriptive approaches by displaying text input and visual input side-by-side, and any modification in one input is reflected in the other. A two-view user interface was first proposed in "Sam," a single interactive system that combines a layout language and graphic modifications for integrated circuits [Trimberger 1981]. Such an interface was soon used by VLSI layout editors [Batali et al. 1981], graphic objects editors [Asente 1989; Nelson 1985], document editors [Brooks 1988; Brooks 1991; Chen 1988], and user interface editors [Avrahami et al. 1989]. Similar to the inefficient machine knitting design and fabrication cycle in Figure 2, "a repetitious cycle of compile, print, walk to the printer, and look" in document debugging was the motive for Lilac [Brooks 1991].

More recently, the two-view approach has been used in interface design [Hibschman and Zhang 2016; Li and Wohlstadter 2008], game development [Unity 2020], graphic drawing [Jacobs et al. 2014; Li et al. 2020], debugging [Guo 2013], output directed programming [Hempel et al. 2019], and understanding program behavior [Lieber et al. 2014].

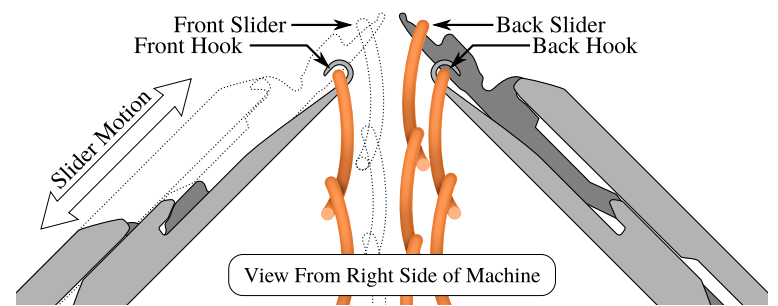

Figure 6: At each position on the bed, a slide-needleequipped $v$-bed knitting machine has four possible loop holding locations: the front and back hooks, and the front and back sliders.

Victor proposed an interface for drawing using JavaScript and HTML Canvas [2012]. One feature of the interface is that if the user rolls over to a line of JavaScript code, the drawings produced by it get highlighted, and if the user rolls over a pixel, it jumps to the line of code that produced it. Inspired by this work, our system offers a similar user experience where if the user selects a line of code, the knitting operations produced by it gets highlighted, and if the user selects a knitting operation, it jumps to the line of code that produced it.

Though our interface does not enable direct visual manipulation like a "complete" two-view approach does, we offer coupling between input and output to achieve the same goal: a clear relationship between the two, which is more than the unidirectional link from input to output in existing CAD software [Kintel and Wolf 2017].

\section{KNITTING MACHINES}

In this section, we provide a brief overview of knitting machines and, in particular, the target machine for our visualization process. For a more general and complete overview, we refer readers to McCann et al. [2016].

Our visualizer targets code written for the Shima Seiki SWGN2 series of knitting machines [Shima Seiki 2014], but the principles used in the visualization extend to almost any modern flat knitting machine. Like other standard v-bed flat knitting machines, the SWGN2 holds yarn on hook-shaped needles arranged into rows along two beds: the front bed and the back bed. Yarn is brought to the needles by yarn carriers that travel between the beds, aligned to the front bed. Each needle can form and manipulate yarn loops on its hook (with the tuck or knit instructions), and pass loops to the hook of the opposite-bed needle across from it (with the $x$ fer or split instructions). The machine can offset (with the rack instruction) the back bed relative to the front bed so different pairs of needles can be aligned; the SWGN2 machines allow a maximum racking in the 4-9 needle range, depending on the machine gauge (needle density). For convenience, we assign each needle an index (running left-to-right) along the machine, and address needles' hooks by their bed and number (e.g., f10 for the hook of the tenth needle from the left on the front bed).

The SWGN2 machines use a needle design called a slide needle [Hull 1927], which - as the name implies - integrates a mechanical slide into the body of the needle. This slide is used during loop 


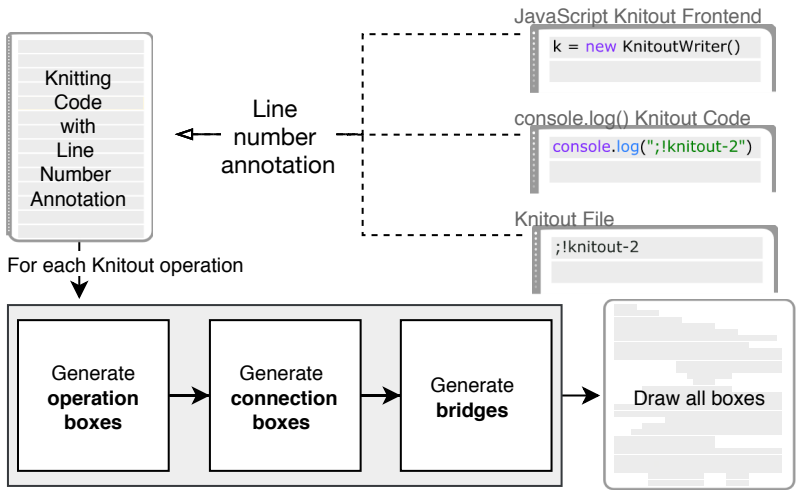

Figure 7: Block diagram of our system. Annotated knitout code generated from the input knitting program file is used to create a box-based visualization coupled to the original file.

formation operations, and can also hold loops independently of the loops held on the hook of the needle. These loops are held "in front" of the loops held on the hook of the needle, and prevent normal knitting operations while they are held. We call these additional loop holding locations the front and back sliders, and refer to them by adding the letter $s$ to their designations (e.g., bs 10 for the slider of the tenth back needle from the left). Thus, for each needle index $\mathrm{N}$, an SWGN2 machine has four loop holding locations (Figure 6): the front hook $(\mathrm{fN})$, front slider $(\mathrm{fsN})$, back slider (bsN), and back hook (bN).

\section{METHOD}

The core of our system is a method for transforming knitting programs into a visualization while maintaining the coupling between them (Figure 7).

Definition 4.1. Operation Box. An operation box is a box directly generated by one of the following knitout operations: knit, tuck, drop, amiss, miss, split, and xfer.

Definition 4.2. Connection Box. A connection box is a box generated to connect operation boxes on the same layer.

Definition 4.3. Bridge. A bridge is a short non-boxed yarn that connects boxes between layers.

To maintain the coupling, our interface connects every knitout operation $^{1}$ generated by a program, to box(es) in our visualization. In order to do this, it transforms the source files into knitting code with line number annotation (Figure 8). For raw knitout files, our interface directly labels each instruction with a line number using a knitout ; ! source: comment. For JavaScript source files, our interface runs the code in an environment with a modified knitout support module and console. log function which adds a ; ! source: comment to any knitout instruction the program emits,

\footnotetext{
${ }^{1}$ Knitout contains a small vocabulary knitting operations - corresponding to basic machine actions - and no variables, loops, or other control flow; this is why it is often generated by a higher-level program.
}

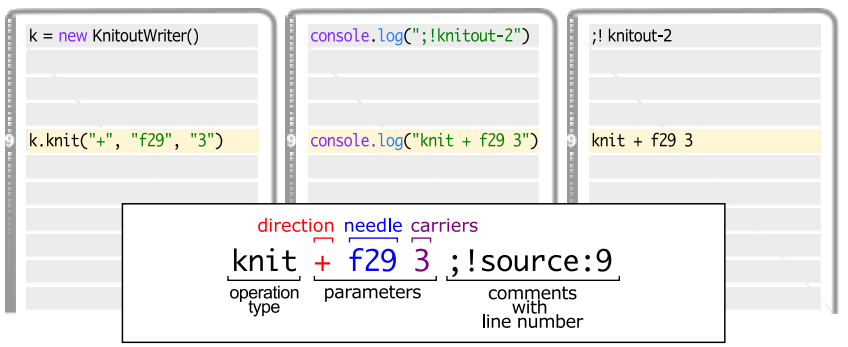

Figure 8: Our interface adds source annotations to knitting code before building its visualization. It supports - from left to right - JavaScript that uses the kni tout module, JavaScript that prints knitout using console. log, and raw knitout files.

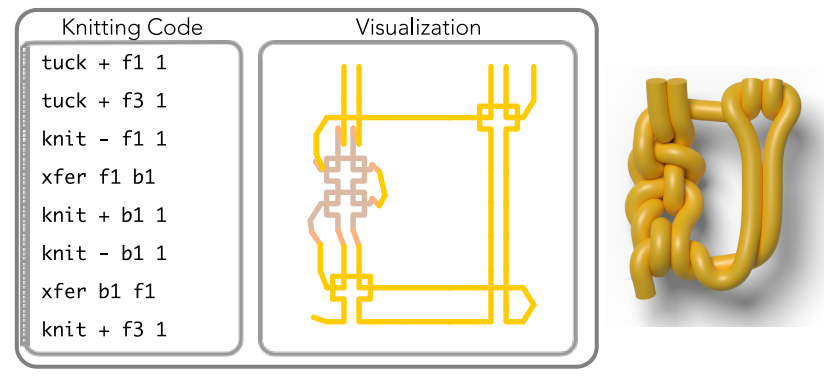

Figure 9: Left: An example of knitting code and its visualization. Yarn in front layers, bridges, and back layers are colored yellow, orange, and brown respectively. Right: Yarn simulation of the same knitting code.

extracting the line number by parsing the a stack trace contained in a JavaScript Error object.

The visualization holds boxes in four layers corresponding to the four types of loop holding locations on the machine: the front hooks, front sliders, back sliders, and back hooks (Figure 6). Each of these layers is further divided into columns, with a column for every needle and a column for every space between needles. The visualizer builds its final image of a knitting program by generating boxes corresponding to machine operations, inserting the boxes into the these columns, and connecting layers with bridges as needed (Figure 9). Once the final image is built, users can locate boxes generated by the selected operations and operations that generated the selected box (Figure 1).

\subsection{Visualization Design Principles}

There are many visualizations one might consider for this purpose. Our selection of a "box-based" topological visualization (Figure 9) - built from operation boxes (definition 4.1) and connection boxes (definition 4.2) - was informed by five principles:

(1) accurate: The visualization must correlate to the knitted output and show distinctions among operations. Our visualization achieves this by illustrating the yarn path(s) in boxes.

(2) fast: The visualization must not take too long to generate. Our visualization has worst-case computational complexity $O(N \times I)$ for $I$ operations and $N$ needles, with the common 


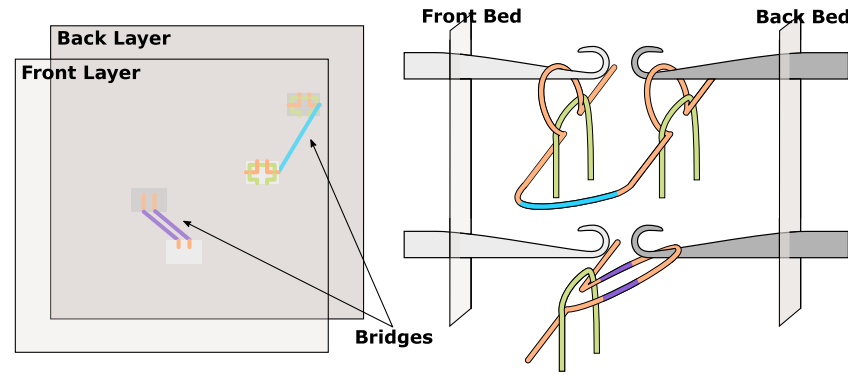

Figure 10: Bridges are short, non-box yarns that connect aligned boxes in the front and back layers.

case being $O(I)$. In practical terms, this means that visualizations can be constructed and displayed at interactive rates (less than a second for reasonably-sized patterns).

(3) chronological: The visualization should show program order. Our visualization achieves this by arranging boxes in first-to-last order from bottom-to-top (as if one were looking at the completed cloth as it left the machine).

(4) bounded-overlapping: The visualization must avoid too many overlapping operations, because this makes it hard to read the output and select boxes. Our visualization places boxes in layers corresponding to knitting machine holding locations (front and back sliders and hooks), so at most two operation boxes and two connection boxes will overlap.

(5) compact: The visualization should take no more space than needed, while remaining readable. In our system, each lowlevel knitting operation generates no more than two operation boxes, and boxes are packed as tightly as possible.

\subsection{Visualization Structure}

The visualization is composed of operation boxes (definition 4.1), connection boxes (definition 4.2), and bridges (Figure 9, 10). Similar to the knitting machine having front and back beds, the visualization has front layers (front hooks and sliders layers) and back layers (back hooks and sliders layers). Boxes are placed in layers, and connections between front layers and back layers are stored as bridges. Thought it is intuitive to think that yarn carriers should have its own layer since it moves between the front and back needles, but in our visualization, yarns move along the front hook layer because, in our target machine, yarn carriers are front-bed referenced (have fixed position relative to the front bed during racking operations).

Layers have columns (Figure 11). Each needle corresponds to a needle column, so all loops made/held on the same needle are in the same needle column. There is space between any two adjacent needles. Each one of these spaces, where yarn travels in between needles, corresponds to a yarn column; all yarn routing between adjacent needles is in the same yarn column. Thus, the number of columns in each layer is determined by the needles used for each bed.

Boxes and bridges are created as the knitout program is parsed. Boxes are placed in columns as soon as the box is generated, but their vertical positions are calculated after all boxes (operation
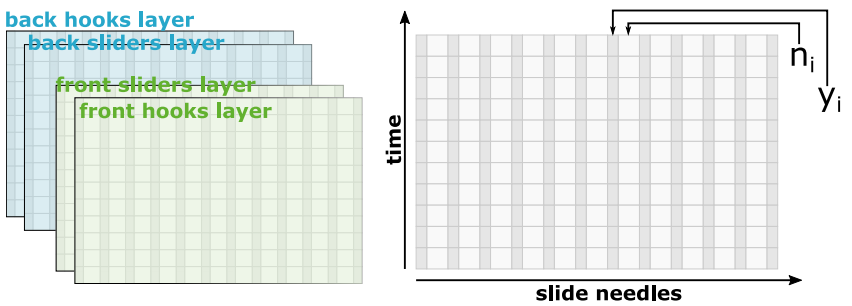

Figure 11: Our visualization is built on four layers, representing the four layers of holding locations on a knitting machine. Each layer is divided into needle columns $n$ and yarn columns $y$ (shaded). $n_{i}$ corresponds to the $i^{\text {th }}$ slide-needle to the right of the leftmost needle in use. $y_{i}$ corresponds to space between $n_{i-1}$ and $n_{i}$.
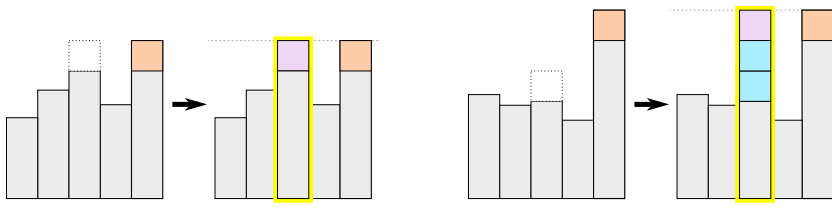

Figure 12: Boxes are placed in non-decreasing chronological order from bottom to top. When a new box (purple) is added, it is never placed lower than a previous box (orange). If (right) a gap would be left in the visualization, connection boxes (blue) are added.

box(es) and connection boxes) are generated for an operation. Vertical positions are determined by their time of construction. The locations of bridges are determined by the boxes they connect.

To ensure compactness (Principle 5), our system places boxes as low as possible while maintaining the program order (Figure 12). It does so efficiently by keeping track of the highest box in each column (and the highest box overall) as the program is parsed, and using these tracked heights to ensure new boxes are placed in non-decreasing chronological (Principle 3) and non-overlapping (Principle 4) order.

\subsection{Operation Box Generation}

All knitout instructions that form loops or move yarn generate operation boxes (definition 4.1). ${ }^{2}$

Specifically, each knit, tuck, drop, amiss, and miss operation generates exactly one operation box. The split and xfer operations move loops from one bed/layer to the other, so generate two operation boxes with one on each layer. Examples of these boxes are shown in Figure 13. For readability, if more than two yarns are used together, our system only shows two of them in the box (Figure 16). For detailed description of each operation, we refer readers to McCann et al. [2016].

In addition to their positioning (layer, column number, height) and content (operation name, yarns used) data, operation boxes also store the line number of the source operation that generated them.

${ }^{2}$ Instructions that configure machine settings, e.g., changing the knitting speed or stitch size, do not generate observable output in our visualization. 

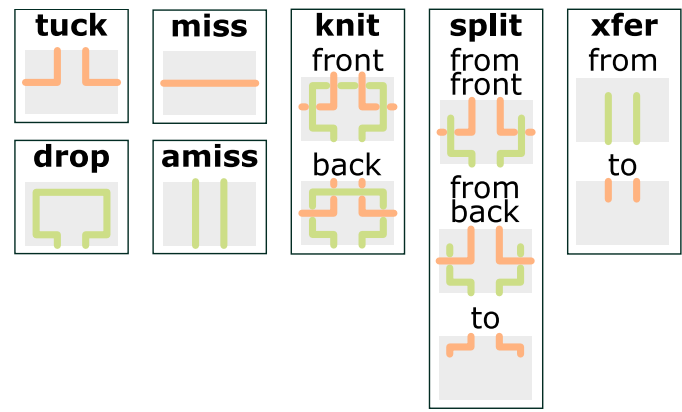

Figure 13: The operation boxes used by our system, shown in their single-yarn variants.
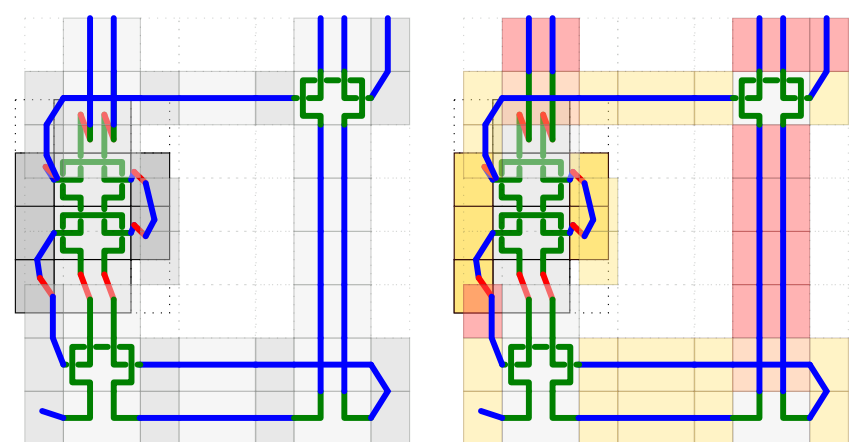

Figure 14: Left: Yarn in operation boxes, connection boxes, bridges are colored green, blue and red respectively. Right: Connection boxes of type (1), type (2), and both are colored yellow, red, and orange respectively.

\subsection{Connection Generation}

In common usage, individual knitting machine operations are performed on the same yarn, and thus are connected by this yarn (if they weren't, the machine would produce a loose confetti of yarn scraps). Thus, operation boxes are connected by connection boxes.

There are two types of connections, of which each connection box can be either or both (Figure 14):

(1) connections between two subsequent operations show the yarn carrier motion along the bed (yellow boxes in Figure 14)

(2) connections between two vertically adjacent operations in the same column join operations over time (red boxes in Figure 14)

In our system, the first type of connection boxes are generated by simulating the movement of the yarn carrier between subsequent stitches (Figure 15). Our system tracks the current position of every in-action yarn carrier as an index into the yarn columns on the front bed, since yarn carriers on the SWGN2 are positioned relative to the front bed. Before every operation, our system visits the column containing the yarn carrier used in the operation and generates yarn connection boxes to direct the yarn toward where it needs to be used (1st column in Fig 15). Our system then visits every column between the current yarn column for the carrier and the

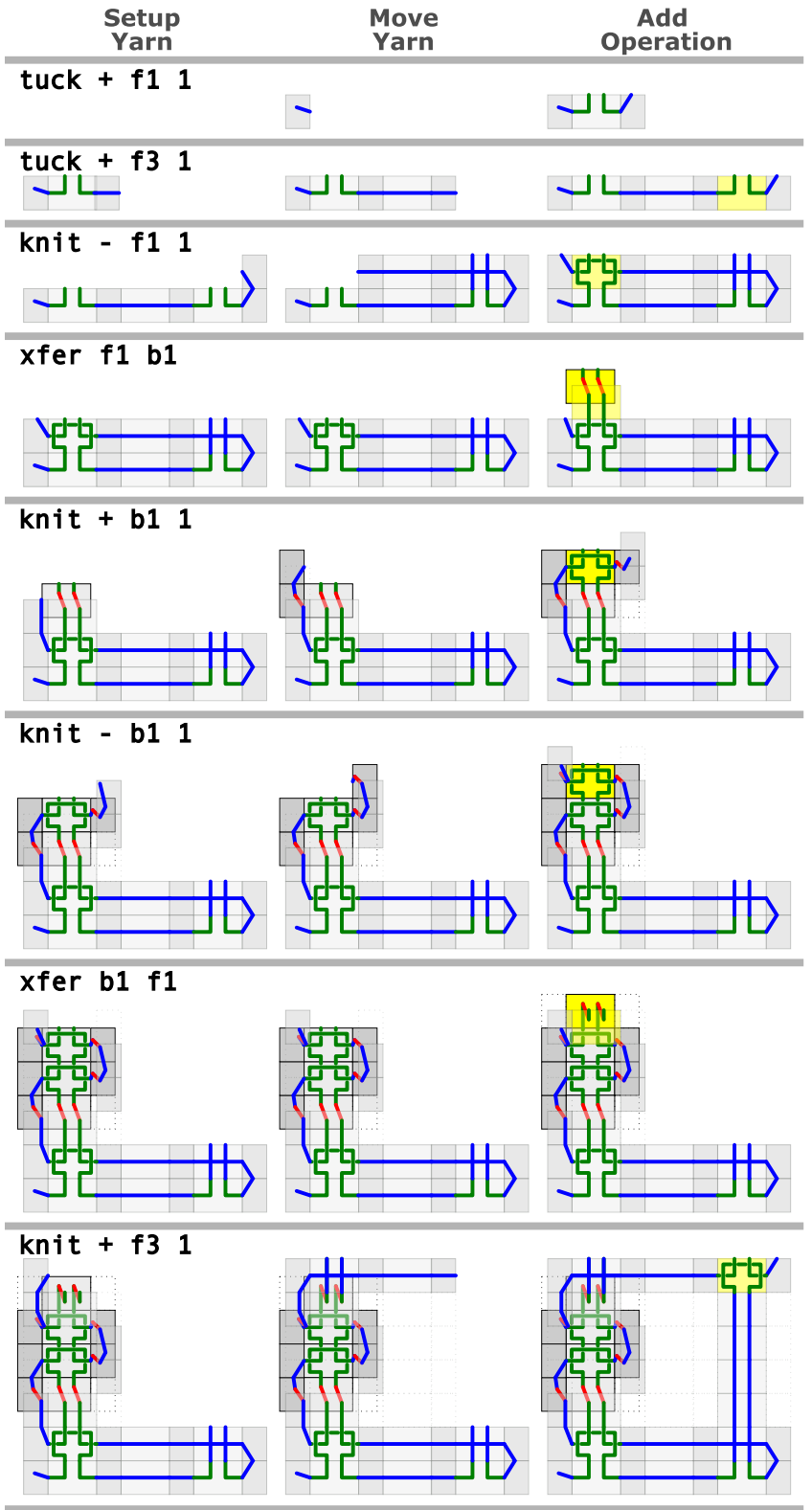

Figure 15: How our system builds a visualization. Each row shows the process of adding a new instruction. First, left, yarn is directed from the yarn carrier toward where (specified needle) it needs to be used (in specified direction); then, middle, connection boxes are added between the current and destination columns; finally, right, operation boxes (yellow highlight) are added and yarn is directed back to its carrier.

yarn column on the front bed just before the operation, and creates a horizontal yarn connection box in each column (2nd column in Fig 15). After the operation, our system creates yarn connection boxes, along with bridges, to bring the yarn to the new current position and direct the ready yarn upwards toward its carrier again (3rd column in Fig 15). 


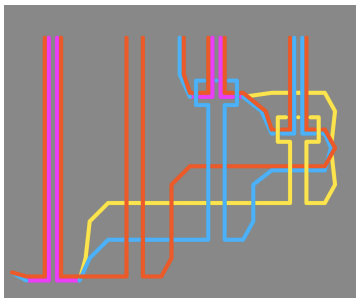

Figure 16: Multiple carriers are in use. The first tuck on the left and the two knits on the right are yarn plating.

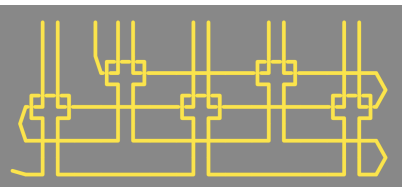

Figure 17: Merged upwardturning and toward-stitchturning yarn connection boxes.

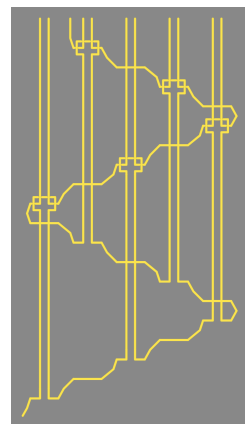

Figure 18: Without merging upwardturning and towardstitch-turning yarn connection boxes.

Type-2 (i.e., column) connection boxes are generated as needed to fill gaps (Figure 12).

It is worth highlighting a few special cases that are important to the functioning of this box-generation scheme. For back bed operations, bridges are added to route the yarn to the back bed yarn column just before the operation and from the back-bed yarn column just after the operation (knit + b1 1 and knit - b1 1 in Figure 15). This is done so that yarn-carrier crossings can be properly represented. In cases where multiple yarn carriers are parked in the same yarn column, appropriate multi-yarn connection boxes are generated to ensure that all yarns parked in the column are available for use at the top of the column (Figure 16). When yarn plating is used, yarn carriers are first moved into the same column, and then moved onward using the method above (Figure 16). In order to prevent long s-shaped runs of yarn, special case logic is used to detect and merge the upward-turning and toward-stitchturning yarn connection boxes (Figure 17, 18).

Connection boxes are generated before and after operation boxes for each operation, depending on the operation. Similar to operation boxes, connection boxes store positioning (layer, column number, height), content data (yarns used), and the line number of the source operation that generated them.

\subsection{Coupling}

After all boxes are generated and the visualization is created from the boxes, we have both code and visualization in the interface. The users can locate the operation that generated selected boxes, and the boxes generated by the selected operation (Figure 1).

The process of locating the operation that generated a box is initiated when the user hovers their mouse cursor over the visualization. Our system finds the box being hovered by converting mouse coordinates to column/row coordinates in each layer, and

checking (front-to-back) for a box with those coordinates. It then visually selects the box by drawing the borders of the box (e.g., white boxes in Figure 24), and finally uses the source code line number attribute of the box to highlight the operation that generated the selected box (e.g., Figure 21, left).

The process of locating the boxes generated by selected operations is initiated when the user selects some lines in the source code. This triggers a redraw of the visualization, wherein all boxes with a source code line number among the selected lines are drawn with a tinted background (e.g., Figure 22, top).
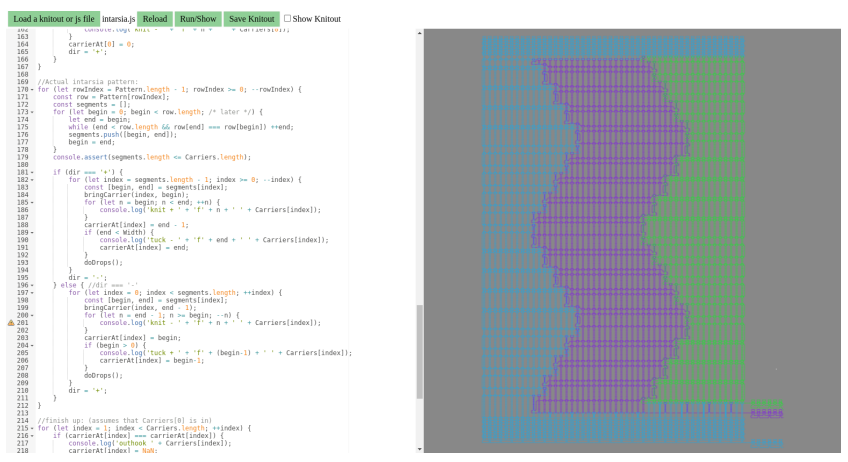

Figure 19: Interface for the visualizing and coupling system. Program and visualization are for the intarsia example.
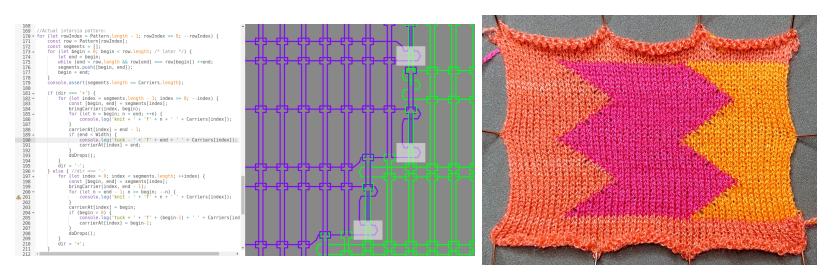

Figure 20: Left: Closeup visualization of tuck operations in the intarsia example. Right: Knitted output of the intarsia example.
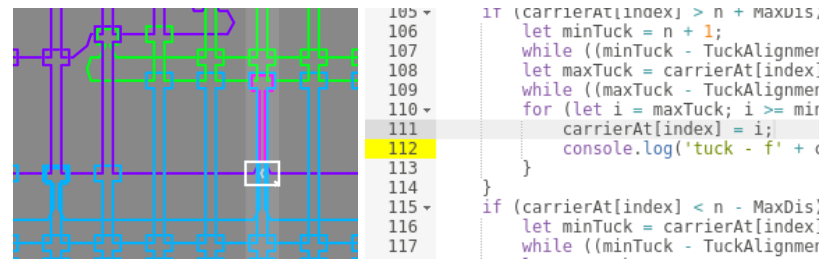

Figure 21: Too many loops tucked on the same needle; a situation that our visualizer helped us catch and fix during the development of the intarsia example. When the user selects the tuck in question (left), the line number of the code that created it is highlighted in yellow (right). 


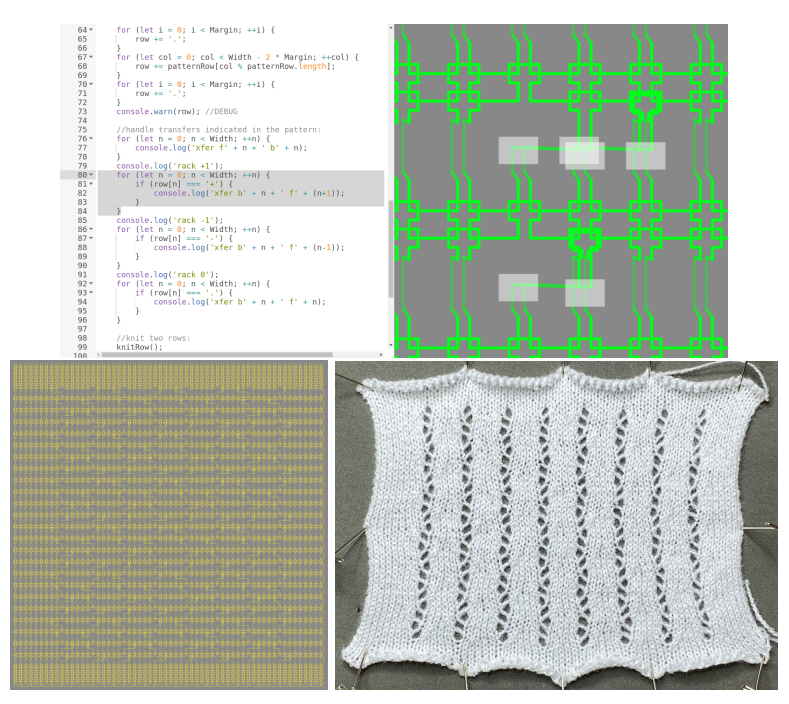

Figure 22: Lace example: Top: Closeup visualization of $x$ fer operations; Bottom left: Visualization; Bottom right: Knitted output.

\section{RESULTS}

The visualization system presented in this paper was implemented ${ }^{3}$ in JavaScript and runs in a web browser (Figure 19). Over the last two years, we have used it both as an aid for prototyping and debugging patterns in an academic lab setting, and as well as a teaching aid in an undergraduate course.

In Figure 20 we present an example of a colorwork technique called intarsia, in which areas of different color are knit in different non-overlapping yarns. It is important in an intarsia pattern to have a strategy to connect the regions of different yarn. In this case, we chose to use a tuck instruction at the end of each row; Figure 20 (left) shows the interface confirming that tucks are being properly placed. In developing this example, our system also helped to find instances where too many yarns were tucking on the same needle (Figure 21), and to find cases where yarn carriers were being moved too far owing to incorrect row directions (Figure 24, top left).

We also created an example of a lace pattern (Figure 22). This example shows how our system is helpful when programming transfer (xfer) instructions, since its diagrammatic presentation makes it straightforward to ensure stitches are moved as expected.

Our system works for large and complicated designs. The "chain link" pattern shown in Figure 23 involves a careful sequencing between all 10 yarn carriers on the system, since the final structure depends on the yarn carriers not tangling during the construction process. Our system was useful for proofreading this interleaving to ensure that yarn was not getting trapped.

As a teaching aid. We have also used this system as part of an undergraduate algorithmic textiles design course, where most students do not have traditional textiles design experience. This tool is used in lectures to explain the mechanics of machine knitting and as part of the suite of tools students use to design and debug

\footnotetext{
${ }^{3}$ https://github.com/textiles-lab/knitout-live-visualizer
}

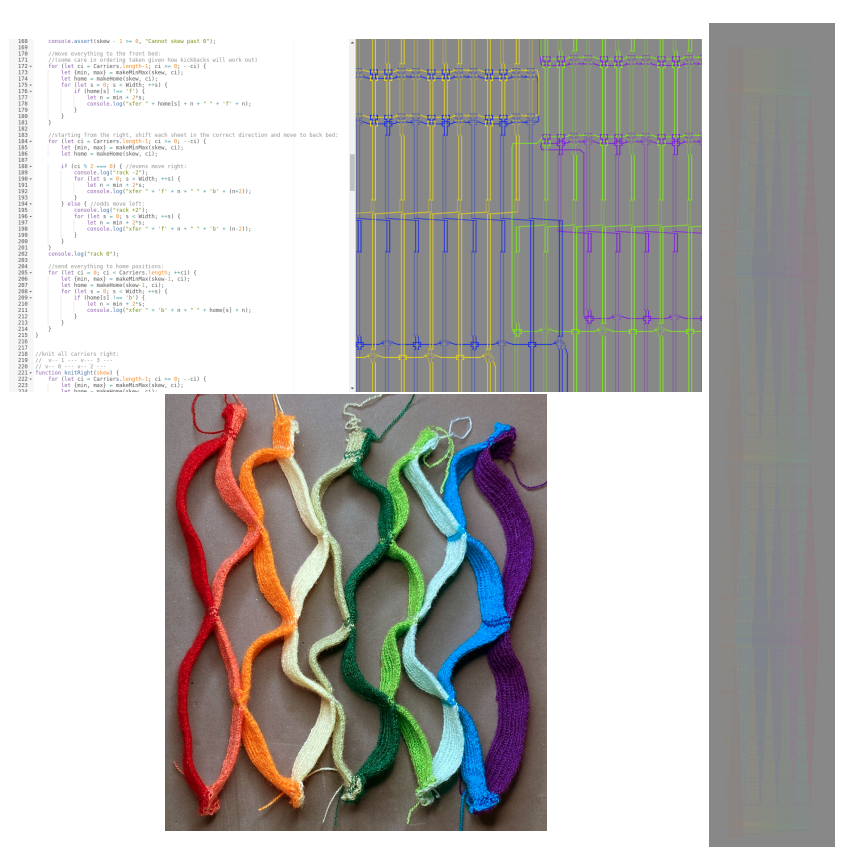

Figure 23: Chain link example: Top left: Closeup visualization of interleaving; Bottom left: Knitted output; Right: Visualization (inscrutable due to the large number of operations).

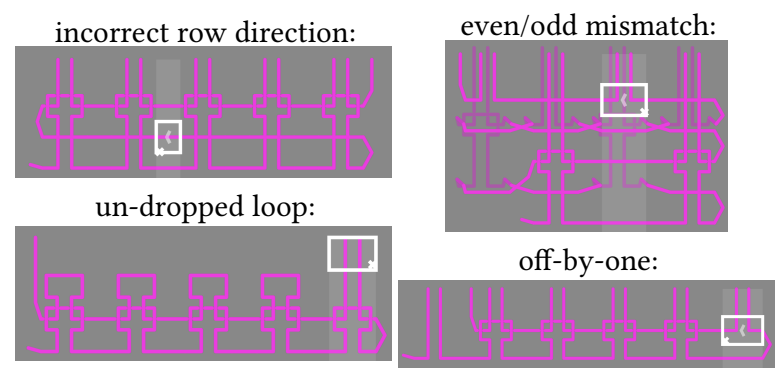

Figure 24: Examples of common errors shown in the visualizer. The visualizer cursor (white box) has been manually placed to highlight the error.

patterns. Our general experience is that the tool helps to catch or at least, explain - a few kinds of common errors (Figure 24) that students make: yarn direction errors, which result in long connecting strands; off-by-one errors, which leave operations misaligned; forgetting to properly drop loops, which leave the loop stretched to the top of the diagram; and even/odd errors, which leave tubes visibly disconnected.

Performance. We evaluated the execution time of our visualizer in a web browser on a workstation computer (Intel Core i7-8086K 4GHz 6-core CPU, 64GB of memory, running Debian GNU/Linux and Chromium 83.0.4103.116). We report in Table 1 the total runtime, the time spent running the user's code and parsing the result (which includes line-number annotation), and the time spent generating 
Table 1: Timings. The parse column includes user code execution, source line number annotation, and knitout code parsing; vis. includes box generation. Instruction counts include only box-generating instructions. Reported numbers are the minimum over 100 runs.

\begin{tabular}{r|rrrr} 
& instr. & parse & vis. & total \\
\hline intarsia & 2681 & $41 \mathrm{~ms}$ & $90 \mathrm{~ms}$ & $131 \mathrm{~ms}$ \\
lace & 6840 & $115 \mathrm{~ms}$ & $161 \mathrm{~ms}$ & $276 \mathrm{~ms}$ \\
chain link & 58320 & $1014 \mathrm{~ms}$ & $3837 \mathrm{~ms}$ & $4851 \mathrm{~ms}$
\end{tabular}

the box-based visualization. For results of a reasonable scale, our visualization can be computed at interactive rates; taking about 2-3x the time of just annotating/parsing the user code.

\section{DISCUSSION}

In creating our knitout visualizer, we chose a specific set of design principles to guide and focus our efforts. These principles were effective in helping us select a visualization that was possible to implement and in creating an understandable system that was useful for debugging topology at the stitch and small-collection-ofstitch level. However, these principles also held the system back, as we discuss below.

First, it is clear that our choice to schematically depict yarn topology results in patterns that are far from predictive of the shape or color of final objects; this is apparent in our lace example (Figure 22) and also clear when doing other common knitting operations like short row shaping and knit/purl patterning. We have already experimented with simple fixes that allow selection of yarn colors via a knitout extension ( $x$-vis-color); one might imagine taking this further and providing a more realistic set of box images. Though, intrinsically, our visualization is limited by the chronological (Principle 3) constraint. Simulation would do a better job of depicting finished yarn shapes, but yarn simulation is much slower than a 2D visualization (Principle 2), and the time order is obscured (Principle 3). Furthermore, yarn can easily tangle and bunch in the simulation, so operations can combine and "smear" into each other which makes selecting/locating a single operation difficult (Principle 4). In the future, it might be interesting to design a visualization that compromises between physical realism and schematic presentation in order to more faithfully match the appearance of the final object while still maintaining readability. Prior works in program state visualizers also provide insights in choosing design principles [Pollock et al. 2019].

By design decision, stitches are always shown with aligned front/back beds and yarn carriers always move relative to the front bed. When knitting on the back bed at non-aligned rackings, yarn looks messy, leaving "unnecessary" yarn paths. This can be alleviated by allowing racking changes dynamically as mouse position changes, or improving the connection box generation logic to prevent such messiness.

Our system's focus on stitch-level depiction also makes it scale poorly to large patterns. For instance, viewing the full scale chain link example at a zoom level that fits the whole pattern on-screen (Figure 23, right) results in individual stitches that are practically invisible. Further, at this operation count, visualizations take a few seconds to compute. However, it is common - especially when experimenting with new techniques - to write knitting programs parametrically, visualizing/debugging/testing at a small scale, and scaling up once the design is finalized and small tests have successfully knit; and our visualization tool works well when designing at these smaller scales. Dealing with tens of thousands to millions of stitches in a readable and understandable way remains one of the great challenges of knit visualization. In simulation, homogenization approaches have been effective [Casafranca et al. 2020; Sperl et al. 2020]; but it is unclear what the topological-visualization analog of these approaches would entail.

Our system's focus on topology means that it does not help with non-topological pattern bugs. For example, while producing the knit lace sample, many stitches were being dropped by the knitting machine because the stitch size was set slightly too small something our interface has no way of showing or understanding. In the future, our system could be refined to flag similar cases by tapping into a crowd-sourced database of common issues and resolutions.

The system not showing or understanding stitch size settings points to another flaw. As explained in Section 4.2, not all knitout instructions generate boxes. Some instructions, like setting stitch sizes, are machine configuration changes. The system could likely be improved by generating boxes for these instructions as well and placing them to the side of the diagram, while maintaining program order.

\section{CONCLUSION}

In this paper, we demonstrated a box-based visualization tightly coupled to a knitting program. We have found that this programming/visualization combination makes it easier to understand and write machine knitting programs by helping to maintain a mapping between instructions and output which would otherwise be lost in current many-step knitting output pipelines.

Though we focused on knitting in this paper, we think that the concept of strongly-coupled visualizations, as well as the design principles behind our visualization, have the potential to be useful in other fabrication domains. Further, we see the problem of maintaining an instruction $\leftrightarrow$ output mapping as fundamental in programming for fabrication, and believe it to be fruitful ground for other software, hardware, and materials-based research interventions.

\section{ACKNOWLEDGMENTS}

Thanks are owed to Vidya Narayanan and Mark Gillespie for their contributions to the development of the system; to Yixin He for prototyping work; to Jenny Lin and Lea Albaugh for feedback on the system; and to all of the users of the knitout visualizer system for their bug reports and suggestions.

\section{REFERENCES}

Harshit Agrawal, Rishika Jain, Prabhat Kumar, and Pradeep Yammiyavar. 2014. FabCode: Visual Programming Environment for Digital Fabrication. In Proceedings of the 2014 Conference on Interaction Design and Children (Aarhus, Denmark) (IDC '14). Association for Computing Machinery, New York, NY, USA, 353-356. https://doi.org/10.1145/2593968.2610490

Paul John Asente. 1989. Editing graphical objects using procedural representations. (1989). 
Autodesk. 2018. Tinkercad.

Gideon Avrahami, Kenneth P. Brooks, and Marc H. Brown. 1989. A Two-View Approach to Constructing User Interfaces. In Proceedings of the 16th Annual Conference on Computer Graphics and Interactive Techniques (SIGGRAPH '89). Association for Computing Machinery, New York, NY, USA, 137-146. https://doi.org/10.1145/ 74333.74347

J Batali, N Mayle, H Shrobe, G Sussman, and D Weise. 1981. DPL/Daedalus design environment (for VLSI).

Kenneth Phillips Brooks. 1988. A Two-View Document Editor with User-Definable Document Structure. Ph.D. Dissertation. Stanford, CA, USA. Advisor(s) Linton, Mark. AAI8826106.

K. P. Brooks. 1991. Lilac: a two-view document editor. Computer 24, 6 (1991), 7-19.

Juan J. Casafranca, Gabriel Cirio, Alejandro Rodríguez, Eder Miguel, and Miguel A. Otaduy. 2020. Mixing Yarns and Triangles in Cloth Simulation. Computer Graphics Forum (Proc. of Eurographics) 39, 2 (2020). http://gmrv.es/Publications/2020/ CCRMO20

Pehong Chen. 1988. A Multiple-Representation Paradigm for Document Development Technical Report. CALIFORNIA UNIV BERKELEY COMPUTER SCIENCE DIV.

Ralph Freshour and Paul Henty. 2016. BenCutLaser CAM program. http://benboxlaser. us/index.php/topic, 614.0.html

Emrecan Gulay and Andrés Lucero. 2019. Integrated Workflows: Generating Feedback Between Digital and Physical Realms. In Proceedings of the 2019 CHI Conference on Human Factors in Computing Systems (Glasgow, Scotland Uk) (CHI '19). Association for Computing Machinery, New York, NY, USA, 1-15. https://doi.org/10.1145/ 3290605.3300290

Philip J. Guo. 2013. Online Python Tutor: Embeddable Web-Based Program Visualization for Cs Education. In Proceeding of the 44th ACM Technical Symposium on Computer Science Education (Denver, Colorado, USA) (SIGCSE '13). Association for Computing Machinery, New York, NY, USA, 579-584. https://doi.org/10.1145/ 2445196.2445368

Brian Hempel, Justin Lubin, and Ravi Chugh. 2019. Sketch-n-Sketch: Output-Directed Programming for SVG. In Proceedings of the 32nd Annual ACM Symposium on User Interface Software and Technology (New Orleans, LA, USA) (UIST '19). Association for Computing Machinery, New York, NY, USA, 281-292. https://doi.org/10.1145/ 3332165.3347925

Joshua Hibschman and Haoqi Zhang. 2016. Telescope: Fine-Tuned Discovery of Interactive Web UI Feature Implementation. In Proceedings of the 29th Annual Symposium on User Interface Software and Technology (Tokyo, Japan) (UIST '16) Association for Computing Machinery, New York, NY, USA, 233-245. https:/doi org/10.1145/2984511.2984570

Joseph Hull. 1927. Knitting-machine needle. https://patents.google.com/patent/ US1634423A

Jennifer Jacobs, Mitchel Resnick, and Leah Buechley. 2014. Dresscode: supporting youth in computational design and making. In Proceedings of Constructionism 2014 Conference. Vienna, Austria, Vol. 10.

Ayelet Karmon, Yoav Sterman, Tom Shaked, Eyal Sheffer, and Shoval Nir. 2018. KNITIT A Computational Tool for Design, Simulation, and Fabrication of Multiple Structured Knits. In Proceedings of the 2nd ACM Symposium on Computational Fabrication (Cambridge, Massachusetts) (SCF '18). Association for Computing Machinery, New York, NY, USA, Article 4, 10 pages. https://doi.org/10.1145/3213512.3213516

Alexandre Kaspar, Liane Makatura, and Wojciech Matusik. 2019. Knitting Skeletons: A Computer-Aided Design Tool for Shaping and Patterning of Knitted Garments. In Proceedings of the 32nd Annual ACM Symposium on User Interface Software and Technology (New Orleans, LA, USA) (UIST '19). Association for Computing Machinery, New York, NY, USA, 53-65. https://doi.org/10.1145/3332165.3347879

Marius Kintel and Clifford Wolf. 2017. OpenSCAD, The Programmers Solid 3D CAD Modeller. (2017).

Jingyi Li, Joel Brandt, Radomír Mech, Maneesh Agrawala, and Jennifer Jacobs. 2020 Supporting Visual Artists in Programming through Direct Inspection and Control of Program Execution. In Proceedings of the 2020 CHI Conference on Human Factors in Computing Systems (Honolulu, HI, USA) (CHI '20). Association for Computing Machinery, New York, NY, USA, 1-12. https://doi.org/10.1145/3313831.3376765

Peng Li and Eric Wohlstadter. 2008. View-Based Maintenance of Graphical User Interfaces. In Proceedings of the 7th International Conference on Aspect-Oriented Software Development (Brussels, Belgium) (AOSD '08). Association for Computing Machinery, New York, NY, USA, 156-167. https://doi.org/10.1145/1353482.1353501

Tom Lieber, Joel R. Brandt, and Rob C. Miller. 2014. Addressing Misconceptions about Code with Always-on Programming Visualizations. In Proceedings of the SIGCHI Conference on Human Factors in Computing Systems (Toronto, Ontario, Canada) (CHI '14). Association for Computing Machinery, New York, NY, USA, 2481-2490. https://doi.org/10.1145/2556288.2557409

James McCann. 2017. The "Knitout" (.k) File Format. https://textiles-lab.github.io/ knitout/knitout.html

James McCann, Lea Albaugh, Vidya Narayanan, April Grow, Wojciech Matusik, Jennifer Mankoff, and Jessica Hodgins. 2016. A Compiler for 3D Machine Knitting. ACM Trans. Graph. 35, 4, Article 49 (July 2016), 11 pages. https://doi.org/10.1145/2897824. 2925940
Vidya Narayanan, Kui Wu, Cem Yuksel, and James McCann. 2019. Visual Knitting Machine Programming. ACM Trans. Graph. 38, 4, Article 63 (July 2019), 13 pages. https://doi.org/10.1145/3306346.3322995

Greg Nelson. 1985. Juno, a Constraint-Based Graphics System. SIGGRAPH Comput. Graph. 19, 3 (July 1985), 235-243. https://doi.org/10.1145/325165.325241

Josh Pollock, Jared Roesch, Doug Woos, and Zachary Tatlock. 2019. Theia: Automatically Generating Correct Program State Visualizations. In Proceedings of the 2019 ACM SIGPLAN Symposium on SPLASH-E (Athens, Greece) (SPLASH-E 2019). Association for Computing Machinery, New York, NY, USA, 46-56. https: //doi.org/10.1145/3358711.3361625

Mariana Popescu, Matthias Rippmann, Tom Van Mele, and Philippe Block. 2018. Automated Generation of Knit Patterns for Non-developable Surfaces. Springer Singapore, Singapore, 271-284. https://doi.org/10.1007/978-981-10-6611-5_24

Shima Seiki. 2011. SDS-ONE Apex3 Design System. [Online]. Available from: http: //www.shimaseiki.com/product/design/sdsone apex/flat/.

Shima Seiki. 2014. WHOLEGARMENT Knitting Machines SWG021N2/041N2/061N2/091N2. https://www.shimaseiki.com/product/ knit/swg_n2/

Georg Sperl, Rahul Narain, and Chris Wojtan. 2020. Homogenized Yarn-Level Cloth. ACM Transactions on Graphics (TOG) 39, 4 (2020).

Hanno Stehling, Fabian Scheurer, and Jean Roulier. 2017. BRIDGING THE GAP FROM CAD TO CAM:: CONCEPTS, CAVEATS AND A NEW GRASSHOPPER PLUG-IN.

Stoll. 2011. M1Plus pattern software. [Online]. Available from: http://www.stoll.com/ stoll_software_solutions_en_4/pattern_software_m1plus/3_1.

Daisuke Suzuki, Tsuyoshi Miyazaki, Koji Yamada, Tsuyoshi Nakamura, and Hidenori Itoh. 2000. A Supporting System for Colored Knitting Design. In Intelligent Problem Solving. Methodologies and Approaches, Rasiah Logananthara, Günther Palm, and Moonis Ali (Eds.). Springer Berlin Heidelberg, Berlin, Heidelberg, 420-425.

B. Swift, A. Sorensen, H. Gardner, and J. Hosking. 2013. Visual code annotations for cyberphysical programming. In 2013 1st International Workshop on Live Programming (LIVE). 27-30.

Jasper Tran O'Leary and Nadya Peek. 2019. Machine-o-Matic: A Programming Environment for Prototyping Digital Fabrication Workflows. In The Adjunct Publication of the 32nd Annual ACM Symposium on User Interface Software and Technology (New Orleans, LA, USA) (UIST '19). Association for Computing Machinery, New York, NY, USA, 134-136. https://doi.org/10.1145/3332167.3356897

S. Trimberger. 1981. Combining Graphics and a Layout Language in a Single Interactive System. In 18th Design Automation Conference. 234-239.

Technologies. Unity. 2020. Unity 2020.1.

Bret Victor. 2012. Inventing on Principle.

Karl D.D. Willis, Cheng Xu, Kuan-Ju Wu, Golan Levin, and Mark D. Gross. 2010. Interactive Fabrication: New Interfaces for Digital Fabrication. In Proceedings of the Fifth International Conference on Tangible, Embedded, and Embodied Interaction (Funchal, Portugal) (TEI '11). Association for Computing Machinery, New York, NY, USA, 69-72. https://doi.org/10.1145/1935701.1935716

Chenming Wu, Haisen Zhao, Chandrakana Nandi, Jeffrey I. Lipton, Zachary Tatlock, and Adriana Schulz. 2019. Carpentry Compiler. ACM Trans. Graph. 38, 6, Article 195 (Nov. 2019), 14 pages. https://doi.org/10.1145/3355089.3356518 